\title{
Test materials for characterising heating from HIFU devices using photoacoustic thermometry
}

Bakaric, Marina, Zeqiri, Bajram, Cox, Benjamin, Treeby, Bradley

Marina Bakaric, Bajram Zeqiri, Benjamin T. Cox, Bradley E. Treeby, "Test materials for characterising heating from HIFU devices using photoacoustic thermometry," Proc. SPIE 11240, Photons Plus Ultrasound: Imaging and Sensing 2020, 112401D (28 February 2020); doi: 10.1117/12.2542429

SPIE. Event: SPIE BiOS, 2020, San Francisco, California, United States 


\title{
Test materials for characterising heating from HIFU devices using photoacoustic thermometry
}

\author{
Marina Bakaric $^{\mathrm{a}}$, Bajram Zeqiri ${ }^{\mathrm{b}}$, Benjamin T. Cox $^{\mathrm{a}}$, and Bradley E. Treeby ${ }^{\mathrm{a}}$ \\ ${ }^{a}$ Department of Medical Physics and Biomedical Engineering, University College London, \\ Gower Street, London, UK \\ ${ }^{\mathrm{b}}$ Centre for Chemical, Environmental and Medical Science, National Physical Laboratory, \\ Teddington, UK
}

\begin{abstract}
High intensity focused ultrasound (HIFU) is a non-invasive thermal therapy during which a focused ultrasound beam is used to destroy cells within a confined volume of tissue. Due to its increased use and advancements in treatment delivery, various numerical models are being developed for use in treatment planning software. In order to validate these models, as well as to perform routine quality checks and transducer characterisation, a temperature monitoring technique capable of accurately mapping the temperature rise induced is necessary. Photoacoustic thermometry is a rapidly emerging technique for non-invasive temperature monitoring, where the temperature dependence of the Grüneisen parameter leads to changes in the recorded photoacoustic signal amplitude with temperature. In order to use this technique to assess heating induced by HIFU in a metrology setting, a suitable test material must first be selected that exhibits an increase in the generated photoacoustic signal with temperature. In this study, the temperature dependence of the photoacoustic conversion efficiency $\left(\mu_{a} \Gamma\right)$ of several tissue-mimicking materials was measured for temperatures between $22{ }^{\circ} \mathrm{C}$ and $50{ }^{\circ} \mathrm{C}$. Materials included were agar-based phantoms, copolymer-in-oil, gel wax, PVA cryogels, PVCP and silicone. This information provided a basis for the development of a volumetric phantom, which was sonicated in a proof-of-concept integrated photoacoustic thermometry system for monitoring of HIFU-induced heating. The results show the suitability of agar-based phantoms and photoacoustic thermometry to image the 3D heat distribution generated by a HIFU transducer.
\end{abstract}

Keywords: Grüneisen parameter, HIFU, metrology, phantoms, photoacoustics, thermometry

\section{INTRODUCTION}

High intensity focused ultrasound (HIFU) is a non-invasive thermal therapy during which a focused ultrasound beam is produced ${ }^{5}$ and an isolated volume of tissue heated above $55^{\circ} \mathrm{C}$ and maintained at this temperature for 1 $\mathrm{s}$ or longer in order to lead to coagulative necrosis and cell death. ${ }^{17}$ The increasing complexity of HIFU sources and treatment delivery regimes means there is an increasing need for model-based treatment planning and more rigorous quality checks. In order to achieve this, a temperature monitoring technique capable of accurately mapping the temperature rise introduced during HIFU in a metrology setting is necessary. The temperature monitoring should be done in real-time with submillimeter spatial resolution and accuracy of at least $1{ }^{\circ} \mathrm{C} .{ }^{6}$

Current imaging modalities used for monitoring the temperature increase induced by HIFU include ultrasound (US) and magnetic resonance imaging (MRI). ${ }^{12}$ These techniques, however, have a variety of limitations including the size of the imaging volume, temperature range, and limited spatial and temporal resolution. Photoacoustic tomography (PAT) is an imaging modality that combines high optical absorption contrast with the high spatial resolution of ultrasound, ${ }^{1,11}$ thus achieving a resolution of $<100 \mu \mathrm{m}$ at a depth of a few centimeters, ${ }^{4}$ as well as high sensitivity and contrast. Photoacoustic thermometry further exploits the temperature dependence of the Grüneisen parameter of tissues or tissue-mimicking materials which leads to changes in the recorded photoacoustic signal amplitude with temperature and can thus be used to determine the temperature of that tissue. ${ }^{15}$

Correspondence: marina.bakaric.16@ucl.ac.uk

Photons Plus Ultrasound: Imaging and Sensing 2020, edited by Alexander A. Oraevsky, Lihong V. Wang,

Proc. of SPIE Vol. 11240, 112401D - @ 2020 SPIE · CCC code: 1605-7422/20/\$21 · doi: 10.1117/12.2542429 
In order to use this technique to assess heating induced by HIFU and perform source characterisation and quality assurance testing, a suitable phantom material must first be selected that exhibits an increase in the generated photoacoustic signal with temperature. The material must also satisfy the requirements for photoacoustic imaging phantoms ${ }^{7}$ HIFU applications ${ }^{17}$ and metrology applications in general. These in short include:

- tissue-realistic, stable and tunable acoustic, optical and thermal properties ${ }^{7,17}$

- strong (and preferably linear) dependence of the Grüneisen parameter on temperature

- high melting point, ideally above $100{ }^{\circ} \mathrm{C}^{12}$

- well-characterised material properties

- durability (longevity) and robustness

- mechanical and optical stability

- reproducibility of fabrication

In this paper, we present the temperature-dependent photoacoustic conversion efficiency for several commonly used phantom materials for photoacoustic and ultrasound imaging, namely agar-based phantoms, copolymerin-oil, gel wax, PVA cryogels PVCP and silicone. This information provides a valuable resource for the further development of a volumetric phantom for photoacoustic tomography imaging of the 3D heat distribution generated by a HIFU transducer.

\section{MATERIALS AND METHODS}

\subsection{Phantom}

The material candidates for the phantoms were characterised in a previously described setup for measuring the temperature dependence of the speed of sound and photoacoustic conversion efficiency. ${ }^{2}$ This study included agar-based materials, ${ }^{9,16}$ copolymer-in-oil, ${ }^{10}$ gel wax ${ }^{13}$ polyvinyl chloride plastisol (PVCP) ${ }^{3}$ and silicone. ${ }^{21}$ The measurement results were verified by characterising degassed deionised water and comparing this to the literature data for the temperature-dependent Grüneisen parameter of water, which was calculated using the approximation $\Gamma(T)=0.0053 T+0.0043$ derived from the definition of the Grüneisen parameter $\left(\Gamma=\frac{\beta c^{2}}{C_{p}}\right)$ and knowledge of the temperature dependence of the speed of sound $c$, volume expansion thermal coefficient $\beta$ and specific heat under constant volume $C_{p}$ for water and aqueous solutions. ${ }^{19}$ In addition, polyvinyl alcohol (PVA) cryogel with dimethyl sulfoxide (DMSO $)^{8}$ as a clearing agent was recently characterised. The fabrication protocols were adopted from the literature, however, for the purpose of this study, acoustic scatterers were omitted and specialised black dyes were added to the base material in order to improve the signal-to-noise ratio. A summary of the base materials and dyes used in the fabrication of the phantoms can be found in Table 1.

Table 1. Base materials and dyes used for the fabrication of the tissue-mimicking materials.

\begin{tabular}{|l|ll|}
\hline Material & Base & Dye \\
\hline \hline Agar-based $^{9,16}$ & $\begin{array}{l}85.27 \% \text { water, 11.21\% glycerol, } \\
3.02 \% \text { w/w agar, 0.5\% potassium sorbate }\end{array}$ & $\begin{array}{l}\text { India ink } \\
\text { (Pelikan Vertriebsgesellschaft } \\
\text { mbH \& Co. KG, Hannover, Germany) }\end{array}$ \\
\hline Copolymer-in-oil $^{10}$ & $\begin{array}{l}78 \% \text { mineral oil, 12\% SEBS, } \\
8 \% \text { LDPE, 2\% BHT }\end{array}$ & $\begin{array}{l}\text { Caligo safe wash relief ink } \\
\text { (Cranfield Colours, Cwmbran, UK) }\end{array}$ \\
\hline Gel wax $^{13}$ & $\begin{array}{l}\text { FF1 003 } \\
\text { (Mindsets Online, Waltham Cross, UK) }\end{array}$ & $\begin{array}{l}\text { Caligo safe wash relief ink } \\
\text { (Cranfield Colours, Cwmbran, UK) }\end{array}$ \\
\hline $\begin{array}{l}\text { PVA/DMSO } \\
\text { cryogel }^{8}\end{array}$ & $\begin{array}{l}\text { (3wt\% PVA 17.4\% water, } \\
\text { }\end{array}$ & $\begin{array}{l}\text { India ink (Pelikan Vertriebsgesellschaft } \\
\text { mbH \& Co. KG, Hannover, Germany) }\end{array}$ \\
\hline PVCP $^{3}$ & $\begin{array}{l}\text { Lure Flex Firm } \\
\text { (Lure Factors, Doncaster, UK) }\end{array}$ & $\begin{array}{l}\text { Black Plastic Color } \\
\text { (Lure Factors, Doncaster, UK) }\end{array}$ \\
\hline Silicone $^{21}$ & $\begin{array}{l}\text { Polytek PlatSil SiliGlass } \\
\text { (MB Fibreglass, Newtownabbey, UK) }\end{array}$ & $\begin{array}{l}\text { Polycraft Black Silicone Pigment } \\
\text { (MB Fibreglass, Newtownabbey, UK) }\end{array}$ \\
\hline
\end{tabular}


The temperature-dependent changes in the PA conversion efficiency of the above-described materials are presented in Fig. 1 (these results were previously presented in Bakaric et al., ${ }^{2}$ except for the PVA/DMSO cryogel). The results are an average of five measurement repeats and are normalised to the amplitude at $22^{\circ} \mathrm{C}$. The coefficients of variation were $<2 \%$ for all tested materials.

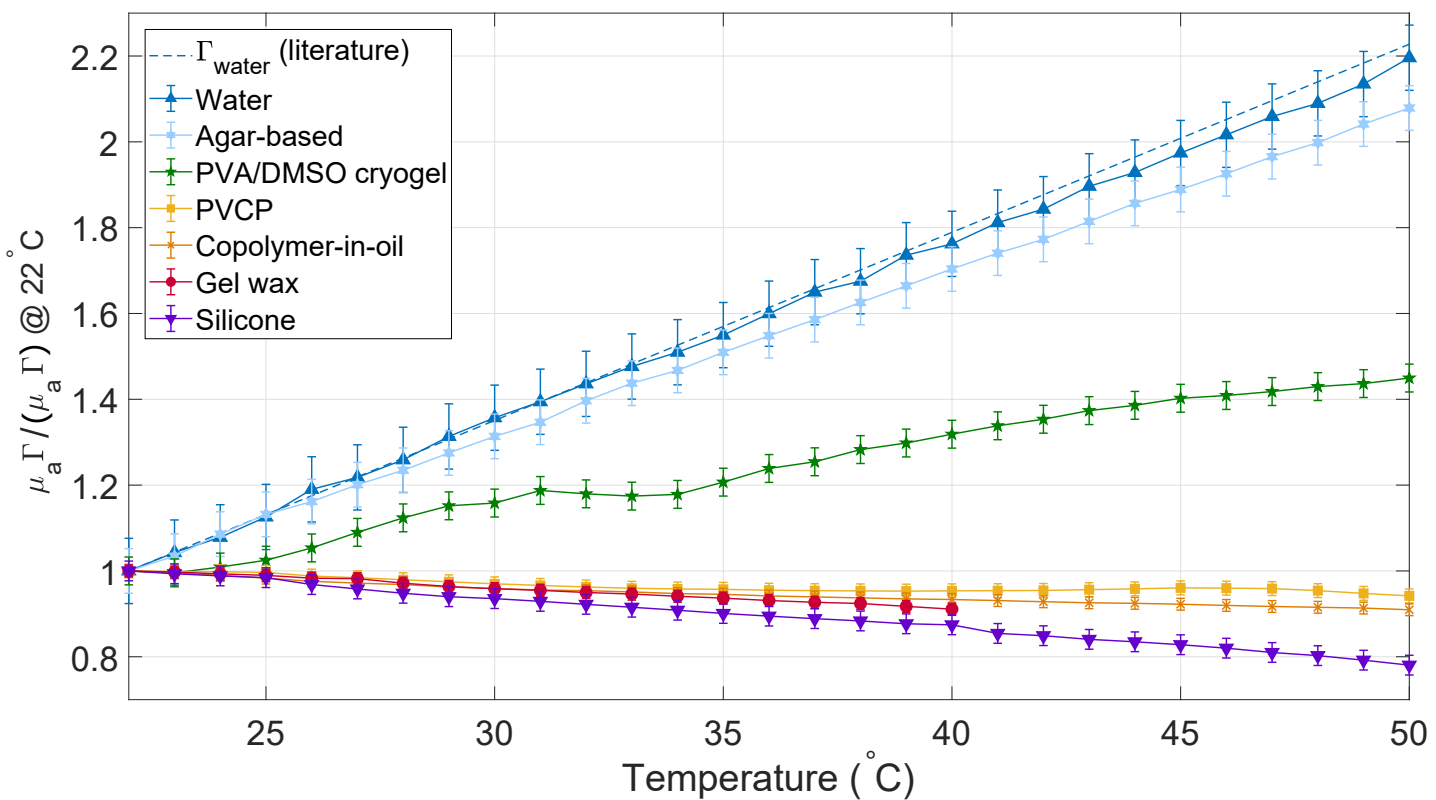

Figure 1. The normalised temperature-dependent change in photoacoustic conversion efficiency $\mu_{a} \Gamma$ generated in material samples over the temperature range from $22{ }^{\circ} \mathrm{C}$ to $50{ }^{\circ} \mathrm{C}$. The errorbars represent the standard deviation between five measurement repeats, with the coefficient of variation $<2 \%$. The literature data for the temperature-dependent Grüneisen parameter of water is presented for reference.

These results show that water-based materials, such as agar-based phantoms and PVA cryogels, exhibit an increase in the PA conversion efficiency with temperature that is dependent on their water content. Copolymerin-oil and PVCP, on the other hand, do not seem to exhibit any significant change in the PA conversion efficiency with temperature. Although their speed of sound decreases by $7-8 \%$ within the measurement range, ${ }^{2}$ the remaining material properties contributing to the Grüneisen parameter and their dependency on temperature are unknown. The photoacoustic signal amplitudes generated in gel wax and silicone samples decrease with temperature. This is expected due to their oil content arising from the base material and dye used, for which the speed of sound is known to decrease with temperature. ${ }^{14}$

Using these results, it can be concluded that the most suitable base materials for photoacoustic thermometry phantoms are water-based. Although PVA cryogels provide better mechanical stability, due to their high acoustic attenuation they are not suitable for HIFU applications. ${ }^{20}$ Thus, agar was selected as a test material and cylindrical phantoms (3 cm in diameter and $4 \mathrm{~cm}$ in length) were fabricated by dissolving $3.02 \mathrm{wt} \%$ agar in $11.21 \%$ glycerol and $85.77 \%$ solution of India ink in water. The most appropriate ink concentration was experimentally determined to be 1 in 13,000, and yielded an optical absorption coefficient of $0.7 \mathrm{~cm}^{-1}$ at $1064 \mathrm{~nm}$ illumination wavelength.

\subsection{Experimental setup}

A schematic of the experimental setup is shown in Fig. 2. The alignment and positioning of all the components was ensured by 3D printing custom-designed PLA holders and mounts (Ultimaker S5, Ultimaker, Geldermalsen, Netherlands). The light delivery (1), HIFU transducer (3) and ultrasound detection array (4) were positioned orthogonally with respect to each other, and the phantom (2.a) was held in a rig holder (2.b) in the centre of the setup. Acoustic coupling was achieved by immersing the entire setup in a tank filled with deionized water at room temperature $\left(22{ }^{\circ} \mathrm{C}\right)$. 


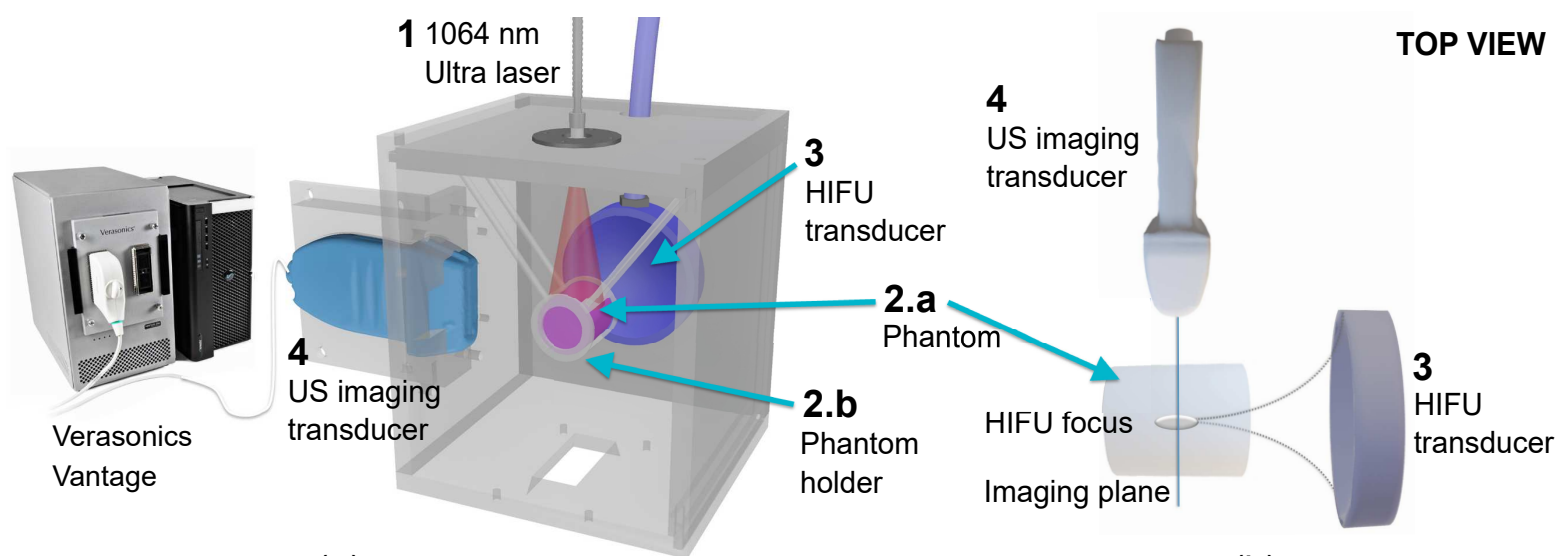

(a)

(b)

Figure 2. Schematic of the experimental setup of the heating experiment. (a) The light from a $1064 \mathrm{~nm}$ Ultra laser was delivered using an optical fibre (1) positioned $6 \mathrm{~cm}$ above the cylindrical phantom (2.a) held in a rig holder (2.b). Phantom heating was achieved using a HIFU transducer (3) with its focus positioned in the centre of the phantom. The generated photoacoustic signals were acquired using a 128 element linear array transducer (4) connected to the Verasonics Vantage 256 Research Ultrasound System. (b) Top view of the setup showing the imaging plane. The HIFU transducer focus and the ultrasound detection array were aligned so that a cross-sectional plane of the HIFU focus was imaged.

The phantom was illuminated from the top using a $1064 \mathrm{~nm}$ Q-Switched Nd:YAG laser (Ultra, Big Sky Laser Technologies, Bozeman, MT, USA). The laser light was delivered using an optical fibre with a numerical aperture of approximately $12^{\circ}(1)$ and positioned $6 \mathrm{~cm}$ above the sample. This spread the beam diameter to approximately $2.5 \mathrm{~cm}$ at the phantom surface and $3.1 \mathrm{~cm}$ at the centre, thus covering the entire phantom. The pulse duration, energy, and repetition frequency were $5.6 \mathrm{~ns}, 40 \mathrm{~mJ}$, and $20 \mathrm{~Hz}$, respectively.

Heating was induced using a single-element spherically focused HIFU transducer (3) (H-101, Sonic Concepts, Bothell, WA, USA) with an active diameter of $64 \mathrm{~mm}$ and focal length of $63.2 \mathrm{~mm}$. The size of the focal volume was $\varnothing 1.26 \mathrm{~mm} \times 11 \mathrm{~mm}$. The transducer was driven at its first harmonic of $1.1 \mathrm{MHz}$ using a burst excitation signal with $50 \%$ duty cycle (25 ms on, $25 \mathrm{~ms}$ off) generated by an arbitrary waveform generator (33500B, Keysight Technologies, Santa Rosa, CA, USA) and amplified by an RF power amplifier (A075, Electronics and Innovation, Rochester, NY, USA) before transmission to the transducer via an impedance matching network (H101G Impedance Matching Network, Sonic Concepts, Bothell, WA, USA). The drive voltage was measured using a scope probe (N2863B, Agilent Technologies, PaloAlto, CA, USA) and an oscilloscope (DSO-X 3024A, Agilent Technologies, PaloAlto, CA, USA). A peak-to-peak drive voltage of $120 \mathrm{~V}$ was used. An acoustic absorber was placed at the rear wall of the tank to prevent acoustic reflections.

The generated PA pressure waves were acquired using a 128 element linear array transducer (4) with a center frequency of $5 \mathrm{MHz}$ and $38 \mathrm{~mm}$ field of view (L7-4, Philips Healthcare, Andover, MA, USA). The imaging plane was a cross-section through the HIFU transducer focus. Data acquisition was carried out using the Vantage 256 Research Ultrasound System (Verasonics, Kirkland, WA, USA) with a sampling frequency of $31.5 \mathrm{MHz}$. The signal acquisition was triggered using an in-house built photodiode. Synchronization between signal receiving and HIFU heating was achieved by delaying the HIFU excitation bursts by $200 \mu \mathrm{s}$ with respect to the laser trigger used for the PA signal acquisition. During the experiments, image acquisition was initiated 10 seconds before HIFU heating was switched on in order to obtain a baseline image at a known temperature. The HIFU transducer was then switched on for 10 seconds, and the gradual heat diffusion imaged for 10 seconds after heating. Acquired data was post-processed offline using Matlab (R2018a, MathWorks, Massachusetts, USA). Images were reconstructed using the kspaceLineRecon function in the $\mathrm{k}$-Wave toolbox ${ }^{18}$ with a standard $2 \mathrm{D}$ linear FFT reconstruction considering an average speed of sound of $1500 \mathrm{~m} / \mathrm{s}$. 


\section{RESULTS AND DISCUSSION}

Figure 3 (a) shows the reconstructed photoacoustic images acquired before (time $=5 \mathrm{~s}$ ), during (time $=15 \mathrm{~s}$ and $20 \mathrm{~s}$ ) and after HIFU-induced heating (time $=30 \mathrm{~s}$ ). HIFU source was switched on 10 seconds after the start of data acquisition and remained on for 10 seconds, after which the data was recorded for 10 additional seconds. The temperature evolution profile as a function of time as recorded by a single channel of the linear array transducer is also plotted for reference in Fig. 3 (b). A steady increase in PA signal amplitude occurs when the HIFU heating is switched on (time $=10 \mathrm{~s}$ ), with the peak occuring after 10 seconds of heating (time $=20$ s). This is followed by a gradual decrease after the HIFU heating is switched off as the deposited heat dissipates from the sonicated region (time $>20 \mathrm{~s}$ ).
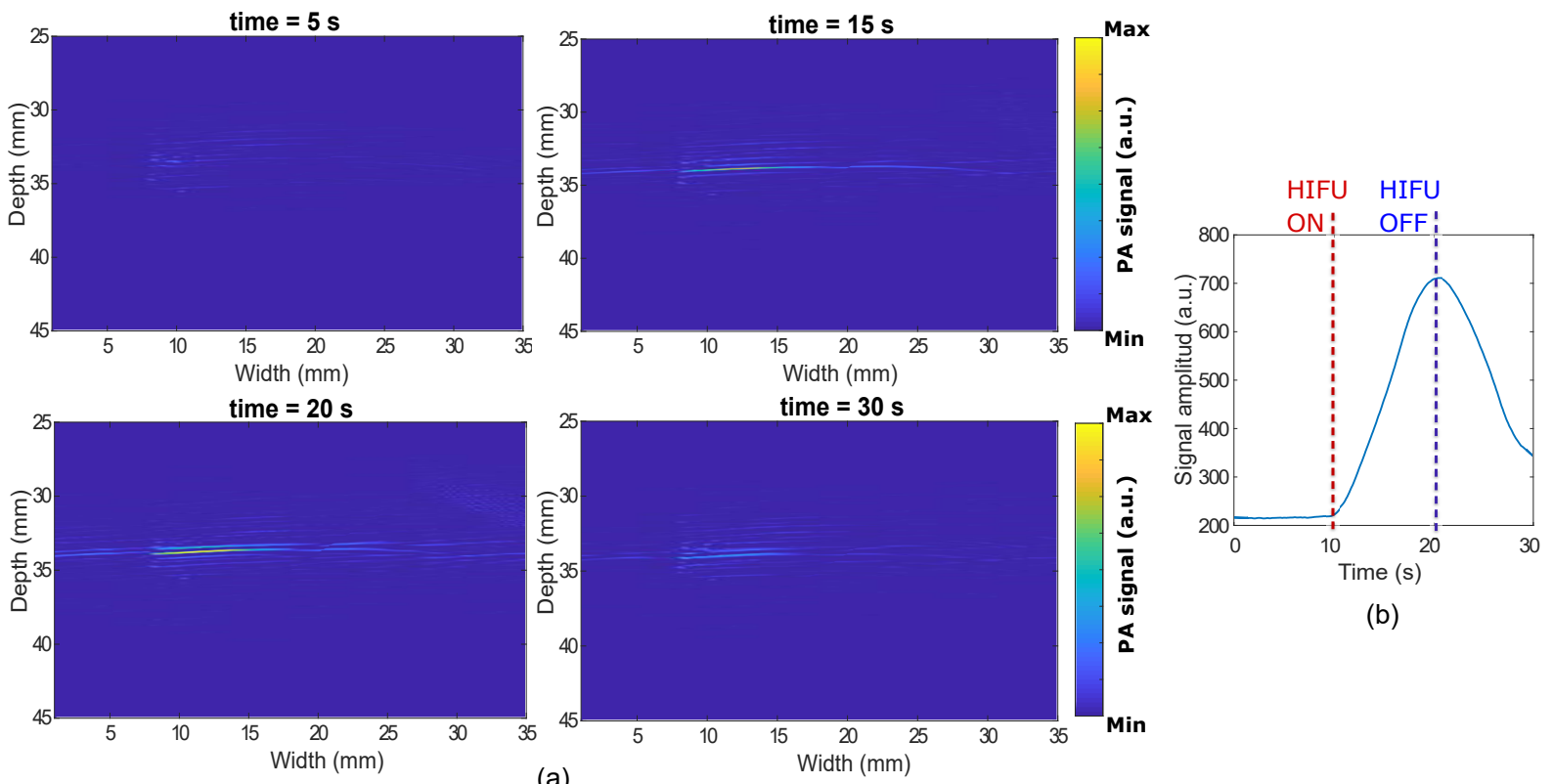

(b)

(a)

Figure 3. (a) Photoacoustic images acquired before (time $=5 \mathrm{~s}$ ), during (time $=15 \mathrm{~s}$ and $20 \mathrm{~s}$ ) and after (time $=30 \mathrm{~s}$ ) HIFU-induced heating clearly show a difference in the photoacoustic signal amplitude for these cases. (b) The temperature evolution profile as a function of time as recorded by a single channel of the linear array transducer. An increase in PA signal amplitude is observed when the HIFU heating is on.

\section{CONCLUSION}

Photoacoustic thermometry provides a potential means of characterizing HIFU devices in a metrology setting, performing quality checks and validating numerical models used for treatment planning. In order to use this technique to assess heating induced by HIFU, stable test materials with suitable optical, acoustic, thermal and photoacoustic properties are required. In this work, the requirements for a PA thermometry phantom were outlined in order to identify the most appropriate phantom materials. The candidates considered for this application included agar-based phantoms, copolymer-in-oil, gel wax, PVA cryogels, polyvinyl chloride plastisol (PVCP) and silicone. Agar was selected as a suitable candidate test material due to its large temperaturedependent change in photoacoustic conversion efficiency. A proof-of-concept integrated HIFU-photoacoustic thermometry system was then developed and used to heat and image an agar-based volumetric phantom. There was a clear change in the reconstructed photoacoustic image in the heated area, demonstrating the potential of characterising heating from HIFU devices using photoacoustic thermometry.

\section{ACKNOWLEDGMENTS}

This work was supported in part by the Engineering and Physical Sciences Research Council, grant numbers $\mathrm{EP} / \mathrm{L} 020262 / 1$ and $\mathrm{EP} / \mathrm{S} 026371 / 1$. 


\section{REFERENCES}

[1] Photoacoustic Tomography. John Wiley \& Sons, Ltd, 2012, ch. 12, pp. 283-321. [Online]. Available: https://onlinelibrary.wiley.com/doi/abs/10.1002/9780470177013.ch12

[2] M. Bakaric, A. Ivory, B. Zeqiri, B. T. Cox, and B. E. Treeby, "Measurement of the temperature-dependent speed of sound and change in Grüneisen parameter of tissue-mimicking materials," in 2019 IEEE International Ultrasonics Symposium (IUS). IEEE, 2019, pp. 1-4.

[3] M. Bakaric, P. Miloro, B. Zeqiri, B. T. Cox, and B. E. Treeby, "The effect of curing temperature and time on the acoustic and optical properties of PVCP," IEEE Transactions on Ultrasonics, Ferroelectrics, and Frequency Control, vol. 67, no. 3, pp. 505-512, 2019.

[4] P. Beard, "Biomedical photoacoustic imaging," Interface Focus, vol. 1, no. 4, pp. 602-631, 2011.

[5] J.-Y. Chapelon, O. Rouvière, S. Crouzet, and A. Gelet, "Prostate focused ultrasound therapy," in Therapeutic Ultrasound. Springer, 2016, pp. 21-41.

[6] R. O. Esenaliev, A. A. Oraevsky, K. V. Larin, I. V. Larina, and M. Motamedi, "Real-time optoacoustic monitoring of temperature in tissues," in Laser-Tissue Interaction X: Photochemical, Photothermal, and Photomechanical, vol. 3601. International Society for Optics and Photonics, 1999, pp. 268-275.

[7] M. Fonseca, B. Zeqiri, P. Beard, and B. Cox, "Characterisation of a phantom for multiwavelength quantitative photoacoustic imaging," Physics in Medicine 83 Biology, vol. 61, no. 13, p. 4950, 2016.

[8] S.-H. Hyon, W.-I. Cha, and Y. Ikada, "Preparation of transparent poly (vinyl alcohol) hydrogel," Polymer Bulletin, vol. 22, no. 2, pp. 119-122, 1989.

[9] I. International Electrotechnical Commission, "Medical electrical equipment: Part 2-37. Particular requirements for the basic safety and essential performance of ultrasonic medical diagnostic and monitoring equipment," IEC Publication, vol. 60601, 2008.

[10] A. M. Ivory, A. Shah, S. Rajagopal, and B. Zeqiri, "Development and investigation of the acoustic properties of tissue-mimicking materials for photoacoustic imaging techniques," in 2019 IEEE International Ultrasonics Symposium (IUS). IEEE, 2019, pp. 1489-1492.

[11] H. Jiang and Z. Yuan, Photoacoustic Tomography. John Wiley \& Sons, Ltd, 2011, ch. 12, pp. 337-367. [Online]. Available: https://onlinelibrary.wiley.com/doi/abs/10.1002/9780470767061.ch12

[12] J. E. Kennedy, "High-intensity focused ultrasound in the treatment of solid tumours," Nature Reviews Cancer, vol. 5, no. 4, pp. 321-327, 2005.

[13] E. Maneas, W. Xia, O. Ogunlade, M. Fonseca, D. I. Nikitichev, A. L. David, S. J. West, S. Ourselin, J. C. Hebden, T. Vercauteren et al., "Gel wax-based tissue-mimicking phantoms for multispectral photoacoustic imaging," Biomedical Optics Express, vol. 9, no. 3, pp. 1151-1163, 2018.

[14] C. A. Miles, G. A. Fursey, and R. C. Jones, "Ultrasonic estimation of solid/liquid ratios in fats, oils and adipose tissue," Journal of the Science of Food and Agriculture, vol. 36, no. 3, pp. 215-228, 1985.

[15] A. A. Oraevsky, R. O. Esenaliev, M. Motamedi, and A. A. Karabutov, "Real time optoacoustic monitoring of changes in tissue properties," Oct. 30 2001, US Patent 6,309,352.

[16] A. Rabell-Montiel, T. Anderson, S. D. Pye, and C. M. Moran, "Attenuation coefficients of the individual components of the international electrotechnical commission agar tissue-mimicking material," Ultrasound in Medicine \& Biology, vol. 44, no. 11, pp. 2371-2378, 2018.

[17] . G. ter Haar and C. Coussios, "High intensity focused ultrasound: physical principles and devices," International Journal of Hyperthermia, vol. 23, no. 2, pp. 89-104, 2007.

[18] B. E. Treeby and B. T. Cox, "k-Wave: MATLAB toolbox for the simulation and reconstruction of photoacoustic wave fields," Journal of Biomedical Optics, vol. 15, no. 2, p. 021314, 2010.

[19] L. V. Wang and H.-i. Wu, Biomedical optics: principles and imaging. John Wiley \& Sons, 2012.

[20] W. Xia, D. Piras, M. Heijblom, W. Steenbergen, T. G. Van Leeuwen, and S. Manohar, "Poly (vinyl alcohol) gels as photoacoustic breast phantoms revisited," Journal of Biomedical Optics, vol. 16, no. 7, p. 075002, 2011.

[21] K. Zell, J. I. Sperl, M. W. Vogel, R. Niessner, and C. Haisch, "Acoustical properties of selected tissue phantom materials for ultrasound imaging," Physics in Medicine 83 Biology, vol. 52, no. 20, p. N475, 2007. 